\title{
Support for Informal Information Use and its Formalization in Medical Work
}

\author{
Charlotte Tang and Sheelagh Carpendale \\ Department of Computer Science, University of Calgary \\ char.tang/sheelagh@ucalgary.ca
}

\begin{abstract}
This paper reports the findings from two field studies that reveal paper-based artifacts being heavily relied upon in medical shift work despite the deployment of a mobile technology in a local hospital ward. We present the information flow in terms of common and personal information spaces and identify three important functions of these personal artifacts in the actual work practices. Artifacts serve as a bedside information source for patient care delivery, an opportune notepad and an information basis for reporting and handover. We thus recommend a system employing digital pens and paper so that nurses can retain the manual practice of constructing their personal artifacts, flexibly use them in the actual work and easily formalize the informal information recorded therein. We also discuss the benefits and challenges of our proposed technology.
\end{abstract}

\section{Introduction}

A hospital is an information-rich environment comprised of distributed information sources. Clinicians often have to physically move during their work to seek necessary medical information which is important for making decisions on issues like diagnosis, treatment and care $[3,5$, 6]. Thus, the tendency towards introducing mobile computing that can provide instantaneous information access at points of care has become prevalent.

We conducted two field studies in a local hospital ward; one before deployment and the other after deployment of a mobile technology. Both investigated the information flow practices during nurses' shift change [12,13]. We observed the construction of informal paper-based personal notes by nurses at the beginning of a shift, both before and after the technology deployment. These personal notes were then carried by the nurses during their shift and served as a frontline information source and an intermediary notepad for information collected during their work. At the end of their shift, they manually disassembled the collected information in their personal notes to various information repositories (Fig. 1). Even after the mobile technology was deployed in our study ward, the paper personal notes

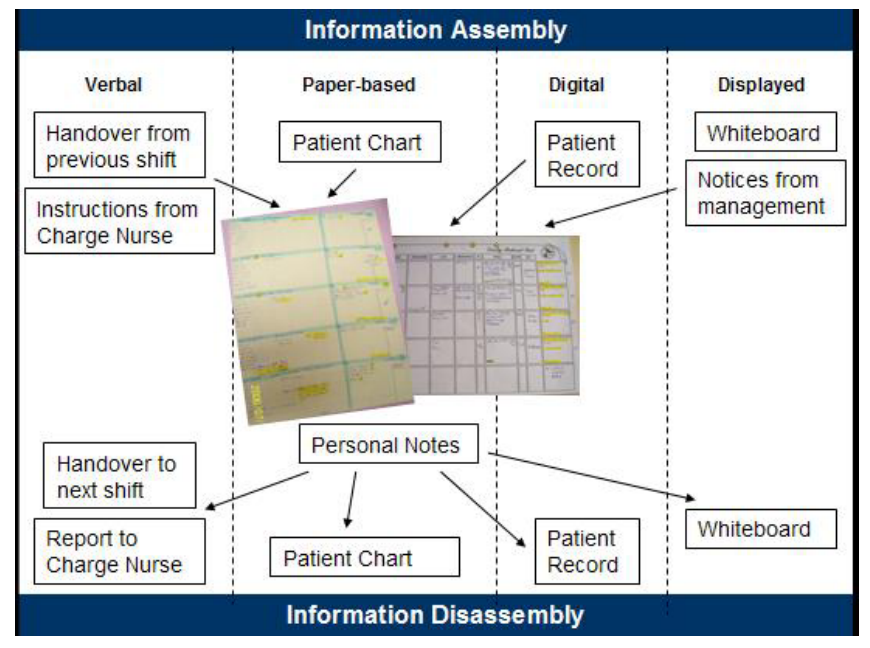

Figure 1. Transition of information through different media types in the information flow process

prepared by nurses continued to play an indispensable role in nursing care delivery; this echoes the findings in Hardey et al., 2000 [6].

Rather than discuss the particulars of the adoption of the mobile technology, our goal in this paper is to present the roles played by the paper-based personal notes in the information flow and how technology should be designed to support these roles. The goal is to design the technology to be as compatible as possible with the practitioner's actual work practices. Nurses in our study ward strongly relied on personal paper notes that they could:

- fold and put in their pockets,

- use to easily and flexibly write, annotate and sketch,

- use to provide an overview of their patients' medical condition, and

- use to construct their work plan for their shift.

In contrast, the electronic health record displayed on the mobile technology screen is rigidly structured allowing only keyboard input and mouse navigation. In addition, the mobile technology itself is quite bulky and awkward to use (Fig. 2).

As paper-based personal notes play such an important and indispensable role in nurses' work, technology should be designed with respect for this work practice $[2,8]$. New technology will likely fail if it hinders work or requires drastic changes to this work practice [10]. It was also found 
that nurses spent considerable time in converting information from their personal notes back to information repositories, primarily the electronic health record (EHR) which is a legal document. Hence, technology should also allow for efficient formalization of informal information recorded in paper personal notes. We argue that the electronic health record should be re-conceptualized to embrace integration with the informal, but indispensable personal artifacts to accommodate both informal use and formal documentation of information.

In this paper, we describe the information flow processes in terms of the conceptual framework of information spaces. Since the information required for accomplishing cooperative work is often spatially distributed, it is important to ensure that information is actively placed and assigned meanings in a common information space where information can be retrieved, interpreted and manipulated for accomplishing and continuing with the cooperative work. A common information space (CIS) "encompasses the artifacts that are accessible to a cooperative ensemble as well as the meaning attributed to these artifacts by the actors" [1,9]. We also defined personal information space (PIS) as an information space that consists of both artifacts and assigned meaning similar to the common information space but is constructed, interpreted and manipulated by only one person [12]. For simplicity, we disregard the mental capacity as part of the PIS in our discussion. Thus we use PIS and personal notes interchangeably in this paper.

In the following sections, we first describe the studies we conducted. We then present the PIS in terms of its roles played in the nurses' work. Next, we describe the dynamics of information flow taking place in nursing care in our study ward. We also discuss how the information flow can be supported by appropriate technology design. We conclude the paper with a discussion on the benefits and challenges of our proposed technology.

\section{The Studies}

We conducted two field studies in a local hospital ward. Our first study, conducted six months before deploying the mobile technology, used minimally-intrusive observations of 42 nurses, interviews and examination of formal and informal information documents to acquire a thorough understanding of the basic information flow practices during nurses' shift change. We reported in [12] that information sharing took place as a pair of parallel processes: information assembly by incoming nurses and information disassembly by outgoing nurses, through four different media: paper, verbal, displayed and digital media (Fig. 1). During the first study, digital information was only accessible through stationary desktop computers. We also identified the information flow between the CISs (e.g., paper-based patient chart and EHR) and the PIS which was typically externalized as paper-based personal notes that

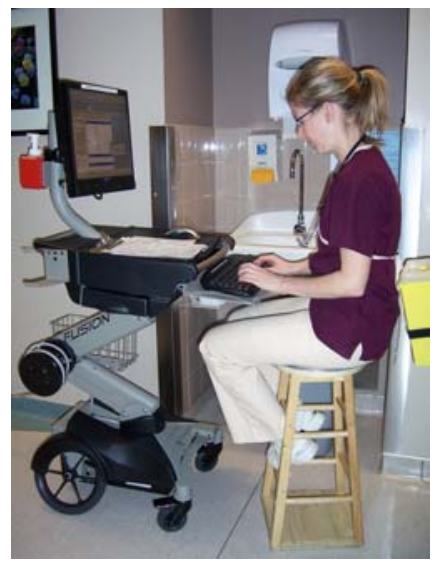

Figure 2. A computer-on-wheel nurses prepared at the beginning of their shift by extracting important information from various information sources. Their shift work depended on the use of personal notes as their intermediate notepad and frontline information source.

Our second study was conducted in two stages, at two and eleven months after the deployment of the mobile technology - computer-on-wheels (COW) - to uncover short- and long-term phenomena. The COW (Fig. 2) is a desktop computer placed on an ergonomically designed mobile cart running on a wireless network to provide mobile access to the EHRs. Methodology was similar to the first study, with the inclusion of an additional questionnaire survey. 38 and 26 nurses participated in the early and later stages of the study respectively and we received 29 filled questionnaires. Despite medical information being pervasively available through the COW, nurses did not change their practice of preparing, carrying and using self-customized paper-based personal notes for their shift work.

In the next section, we focus on the PIS and its crucial role in the nurses' work. We characterize this information space in terms of its functions in nursing care.

\section{Personal Information Space}

We identified three important functions of the personal notes in terms of their roles in nursing care in our study ward. First they serve as a bedside information source and work plan for patient care delivery. The artifacts are portable and malleable and provide customized information at the point of care. Second they serve as an opportune notepad where nurses may flexibly scribble notes and annotations. This is important as new data emerges frequently and ubiquitously. Third, they are the information basis for reporting and handover. Information added to the physical artifacts during a shift often forms part of a patient's illness trajectory. Therefore such information must be properly documented for use by other clinicians and for later review when needed.

\subsection{Bedside information source and work plan}

Personal notes that we collected in our studies exhibited both a high degree of personalization (see Fig. 1) in how the information was represented and many similarities in the kinds of information extracted from various information 
sources (e.g. patient chart, EHR). In general, they contain both static and dynamic data. The static information is primarily identification information such as patient demographics and their medical history. The dynamic information includes medical information such as vital signs, medications and test results directly extracted from various medical records and recorded during the day as well as reminders and to-dos, alerts, prompts, scheduling, verification and reporting information. However, an examination of their presentation and representation, with personalized augmentations, informs richer meanings than plain medical facts. For instance, complex and highlypersonal augmentation practices were used with information to indicate different levels of importance or urgency, or to facilitate information retrieval. These augmentations included specific spatial arrangement of information, physical attributes of the personal notes and various visual augmentations used to enhance the information and its functions (e.g., Fig. 3a \& 3b). The information is presented in such a way that it also served to guide specific actions. Details of these information types and augmentations can be found in [12].

\subsection{Opportune notepad}

As described in Section 3.1, personal notes were prepared as the immediate source of medical information source, and the plan for carrying out nursing work. When information was placed in personal notes, nurses usually organized in their mind what and when their tasks would need to be done and what new information would emerge during their work shift. Therefore they often made liberal use of information holders such as text lines and checkboxes in addition to the more conventional reminders and to-do lists. These information holders served as obvious memory triggers by prompting the nurse who prepared the personal note to carry out the specified task and to fill in the required information or task status (Fig. $3 b$ ). On the other hand, unanticipated information may emerge especially for unstable patients. Thus nurses would always improvise their personal notes to accommodate the unexpected yet important medical information. Nurses often first recorded the emerging information in their personal note as a means to reduce their mental workload and eventually reported in official documents of varying media. As most personal notes were pre-structured for specific kinds of information, nurses usually just casually added the unforeseen information in any available open space not specified in the usual spatial layout of their personal note.

\subsection{Information basis for reporting and handover}

Our classification of information found in personal notes included a reporting type which is an umbrella term for all the information recorded in the personal note that must be reported or documented so that other clinicians can use for their decision making. Such information includes qualitative assessments, quantitative measurements, changes in treatment and care plan, and new medications prescribed. The current formalization process required nurses to manually convert the recorded information into report format using definite terminology and language.

\section{Dynamics of Information Flow}

During our study we observed the use of both CIS and PIS in the information flow process. In the following we describe how information flows from the CISs to the PIS, how a PIS is actively used to support the shift work, and how information is finally converted from the PIS to the CISs. Our description illustrates how the creation, use and retirement of a PIS plays a vital role in nursing care.

\subsection{From CIS to PIS}

To facilitate work, nurses often convert information found in CISs of different media to paper-based medium at the beginning of their shift (Fig. 1). They manually write down different kinds of information in designated locations on to their personal note, augmented with an array of spatial and visual attributes [12]. Many nurses commented that the manual transposing of mundane information such as patients' demographics is time-consuming and would welcome a paper template pre-loaded with the static information.

\subsection{Use of PIS}

We identified how the PISs were maintained and dynamically altered during shift work from the traces left in the personal notes that we collected and from interviews with the nurses. Nurses generally used a different-colored pen for additions to their PIS. They relied heavily on their PIS for necessary medical information relating to their patients, only consulting the EHR or patient chart when the required information could not be found in the PIS. They wrote down new information immediately on their PIS at points of care. Depending on the nature of the gathered information, they might use different visual cues (e.g., an asterisk or a different color) to signify its importance or urgency, much in the same way they augmented information when they first constructed the PIS. In short, nurses actively used the PIS as an instantaneous information source and as a convenient notepad to temporarily record information.

\subsection{From PIS to CIS}

Information flow across shifts is achieved by outgoing nurses packaging and placing the information (with intended meaning) in appropriate CISs so that the 
information can be shared with concerned personnel. Outgoing nurses often relied on the same personal note that they used and added new information to during their shift.

Placing information from a PIS to the CISs (i.e. charting) is time-consuming (noted in observations and questionnaires). Nurses needed to dispense information to specific CISs depending on the nature of information gathered. For example, outgoing nurses needed to compile shift-specific nursing care information for their incoming colleague, provide a high-level patient's state to their charge nurse, write important treatment- and medicationrelated information in patient charts for responsible physicians and navigate the highly structured and deeply hierarchical EHR to document a formal nursing care record. In practice, nurses often use a combination of physical artifacts and their mental capacity as the constituents of their PISs [12]. As most of the information transferred to the CISs could be found in the PIS, this transposing activity is generally considered redundant and time consuming [4]. Thus a system that allows efficient transposing of information between the information spaces could improve patient care as nurses could then spend more time with their patients rather than, for example, sitting in front of a computer juggling information between the information spaces.

\section{Initial Design Scheme}

Our analysis on how personal notes were constructed and used indicates that the personal notes play a central role in the nurses' work. Replacing it with a mobile digital device such as a PDA may not provide nurses with the same affordances offered by paper artifacts as described in Section 3. Thus our initial design focused on facilitating the preparation of the PIS. For instance, a system that allowed nurses to easily move (e.g., drag and drop) information from the EHR directly onto a personal note template might be useful to the nurses. The personal note template would be pre-loaded with static patient information and could be customized to suit each individual's preferred spatial arrangement of information, visual representations and markups such as highlighting, font color, special icons, text lines and checkboxes. The template could then be printed in color as their personal note for use during their shift in a similar manner to that which they were familiar.

We informally presented our design idea to some of the nurses working in our study ward. Our goal was to obtain feedback for refining our design. However we received unanimously negative response from the nurses who, quite unexpectedly, favor manually writing down medical information themselves. However, they still desired having the static information pre-printed on their personal note sheet. The manual handwriting exercise, as they explained, actually helped them remember their patients' condition and the tasks that they were required to perform on/with them since the manual activity helped build a mental map of their work plan. Therefore having an electronic system where they only read the medical information, then transferred information onto a template via mouse manipulation would prevent them from building a conceptual work plan. As such, we understood that this manual writing practice was an essential part of the shift change activities that should be respected with the introduction of technology. After all, technology should be designed to support actual work practices [14].

\section{Revised Design Concept}

Feedback from our preliminary design idea clearly indicated that a system should be designed to support the work practices familiar to the nurses. Therefore our redesign rationale strives to accomplish the following. The system should:

- retain the practice of manually transposing information from the CISs to a paper-based PIS,

- allow flexible use of a paper artifact to support and enhance work performance, and

- allow users to effortlessly move information from the paper artifact back to the CISs in order to provide timely information and to reduce charting time.

\subsection{The technology}

We propose the use of a digital pen and smart paper technology such as Anoto technology [15], equipped with handwriting recognition software. The digital pens are similar to ordinary pens in that they use regular ink, but different in that they include a built-in camera which continually scans the paper as ink is applied. All pen strokes are captured and stored in memory for later transmission either through a Bluetooth technology or via a docking platform. The smart paper is like ordinary paper, but is pre-printed with specially arranged dot patterns which uniquely define the absolute coordinates on the paper. Templates for spatial layout of information may be specified to create artifacts such as information holders or storage bins [11] that may be assigned for specific kinds of information which in turn can be mapped to corresponding fields in the digital database. Such design allows efficient information transfer from the smart paper to the digital medium. Also, a handwriting recognition can process specified symbols into digital characters which can then be stored in the EHR database. In addition, an exact digital replica of the personal note can be optionally captured and saved for future review.

\subsection{Interplay of information spaces}

Figure 3 illustrates our design concept. In our proposed system, nurses will use a digital pen just the way they have been using pens for writing. The smart paper can be customized for individualized layout of information 


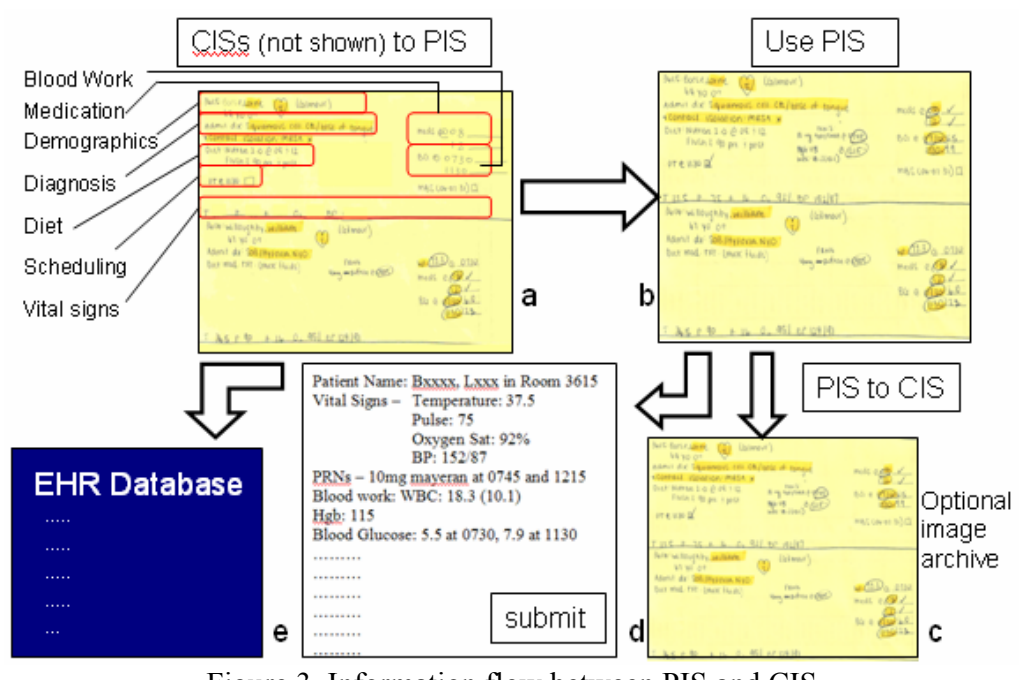

Figure 3. Information flow between PIS and CIS

Thus, our findings urged us to take a closer look at the roles played by these personal notes, how and why they are vital to nurses' work and how we can design technology to support their roles. We identified their importance as a bedside information source for patient care delivery, an opportune notepad and the information basis for reporting and handover. We therefore formulate our design rationale to support these roles. Nevertheless, our initial design idea assumed that nurses would prefer digital solution in all aspects of their work context, without considering the deeper impact of the manual exercise. We thus learnt that it is important to also understand the underlying effects of all facets of their activities in order to design for supporting their work practices.

As such, the use of a digital pen and smart arrangement which has shown to be important for efficient information retrieval (Fig. 3a). Static information will be preprinted on the smart paper; whereas dynamic information will be assigned to information holders or storage bins. For example, all the blood work results will be written in the storage bin assigned for "Blood Work", similarly for the "Vital Signs" bin. They can also augment information in their personal note in the manner to which they are accustomed. As a result, nurses can construct their PIS, retaining the manual activity which helps them build a mental map of their shift work.

During the shift, nurses can flexibly use the personal note for annotation or sketches as in their current nursing practice. Ink strokes captured in the digital pen's memory are readily downloadable via either Bluetooth or docking technology. The captured ink strokes can be converted by handwriting recognition software to digital characters as appropriate for the EHR (i.e. PIS to CIS). Recorded information in PIS can be optionally archived in the database as an image file of the personal note (Fig. 3c) as a back-up information source for later review. The converted information can then be displayed with appropriate labels corresponding to the fields to be stored in the EHR. Nurses may alter, add or delete information from this record (Fig. 3d). The verified version can then be submitted for archiving in the EHR database (Fig. 3e). In this way, nurses can, in a simple and timely manner, convert handwritten information recorded in paper medium into digital information for distributed use by other clinicians.

\section{Strengths and Challenges}

We repeatedly found the importance of personal notes in nurses' work practice in our study ward before and after the deployment of the mobile computing device which was intended for ubiquitous use at points of care. While many organizations invest handsome amounts of money in largescale innovative technology, most do not pay enough attention to the actual work practices of their employees.

paper might best benefit their work practices. Nurses can continue their shift preparation practice using the apparently traditional pen and paper. This activity helps them build a strong mental model of their shift work. The use of their personal note as an immediate information source and opportune notepad can also be achieved. In the mean time, understanding the importance of spatial arrangement of different kinds of information allowed us to use the information holders or storage bin concept to map to appropriate fields in the EHR database. This set up permits efficient and timely updates of medical information in the digital medium which is frequently and remotely shared by an array of clinicians. Thus our system is designed to support nurses as they carry out their work. Ideally it should also help lower time cost which is likely influential in view of the severe nurse shortage currently experienced in the local health region. We also value the personal aspect of the artifacts as some information may not be desirable to be archived for review. Thus the image capture of personal notes is designed to be optional.

In general, our proposed technology can potentially offer a bridge between paper and digital media. Therefore, information recorded in paper-based patient charts which are still heavily in use in our study ward, mostly by physicians to record their notes, can easily be digitized for distributed use by clinicians. Currently, clinicians have to physically go to the centrally-located nursing station to retrieve information from these charts which unfortunately are often misplaced or removed from their designated locations [12]. Therefore information flow between a CIS (e.g., paper patient chart) and another CIS (e.g., EHR) can also be improved with this use of digital pens and smart paper.

There are still challenges that need to be overcome when implementing our proposed system. First, current practice involves heavy use of free-form annotations on the personal notes. The use of information holders or storage bin concept sets the boundary in which specific kind of 
information may be scribbled and correctly recognized by the system. Hence nurses' handwriting practice may have to alter somewhat to ensure that specific data does not fall outside the storage bin boundaries.

Second, the handwritten notes will be processed by handwriting recognition software which unfortunately is not very mature at the present and thus may produce unexpected conversions. Again, nurses may have to adjust their writing habit by writing more legibly. Yet this is not always possible especially during critical situations when they can only manage to quickly scribble down required information. Fortunately, our design provides for a verification step to ensure correct information is formalized properly in the database. Possible drawback of this is that nurses may find it too heavy-weight to verify the information before archiving, so defeating the purpose of effortlessly updating medical information in the digital medium. However, even with this verification it should be simpler that the current manual entry.

Third, special icons, signs and terminology are commonly used in recording medical information in personal notes. Examples include @, *, ‘, Q4H (every 4 hours), SOB (Short of breath) and v/s (vital signs). They are important for nurses to more efficiently record gathered information. Our proposed system will therefore be required to recognize and translate them correctly.

Fourth, although we have identified a generally consistent set of information types that nurses placed in their personal notes, out-of-ordinary information is sometimes scribbled in arbitrary spatial locations. We recommend that such information may be handled in two ways. The system may disregard information placed in locations outside the boundary of any storage bins such that those spaces will be used for more informal scribbling of notes. As such, these spaces would be ideal for placing information that nurses do not want to be captured and accidentally archived in the digital medium. Personal stereotypical comments, e.g., "this patient is difficult!" is an example of such information. Alternatively, the system may consider all the spaces outside the boundary of storage bins as one big storage bin. For instance, there can be an information holder or storage bin for miscellaneous information such that all data in this bin will be captured and processed for manual classification at the verification stage. Nurses can then re-arrange the collected information for archiving in appropriate fields of the EHR database.

Finally, while the smart paper is regular paper with unique dot pattern imprinted, digital pens are much more expensive technological device than ordinary pens. Nurses do not normally exercise care when using a regular pen. They do not worry if it drops on the floor or is accidentally left behind. But with an expensive digital pen which nurses may also have to be personally responsible for, they will have to handle it with prudence to ensure their proper functioning and to reduce the replacement cost for lost or damaged devices. In addition, handling of a pervasive digital device at work may also have an impact on their psychological perception. Thus a formal study may need to be conducted to evaluate the impacts of our proposed system may have on the well-being of the nurses and their work practices.

\section{Acknowledgements}

We thank the nursing staff on the W21C at the Foothills Hospital for their incredible support. We also thank our sponsors: AIF, iCore and NSERC for their generosity.

\section{References}

[1] Bannon, L. and Bodker, S. Constructing Common Information Spaces. In Proceedings of the ECSCW, 1997, Kluwer, pp. 81-96.

[2] Bardram, J. and Bossen, C. A Web of Coordinative Artifacts: Collaborative Work at a Hospital Ward. In Proceedings of GROUP 2005, pp. 168-176.

[3] Bardram, J. and Bossen, C. Mobility Work: The Spatial Dimension of Collaboration at a Hospital. In Journal of CSCW, 14(2), pp. 131-160.

[4] Cabitza, F., Sarini, M., Simone, C. and Telaro, M. When Once Is Not Enough: The Role of Redundancy in a Hospital Ward Setting. In Proceedings of GROUP 2005, pp. 158-167.

[5] Gonzalez, V., Tentori, M., Moran, E., Favela, J. and Martinez, A. Understanding Mobile Work in a Distributed Information Space: Implications for the Design of Ubicomp Technology. In Proceedings of CLIHC 2005, pp. 52-63.

[6] Hardey, M., Payne, S. and Coleman, P. 'Scraps': Hidden Nursing Information and its Influence on the Delivery of Care. In Journal of Advanced Nursing, 2000, 32(1), pp.208214.

[7] Luff, P. and Heath, C. Mobility in Collaboration. In Proceedings of the CSCW 1998, pp. 305-314.

[8] Luff, P., Heath, C., and Greatbatch, D. Tasks-in-Interaction: Paper and Screen Based Documentation in Collaborative Activity. In Proceedings of CSCW 1992, pp. 163-170.

[9] Schmidt, K. and Bannon, L. Taking CSCW Seriously: Supporting Articulation Work. In CSCW: An International Journal, Vol. 1, No. 1, 1992, pp. 7-40.

[10] Scott, S., Grant, K. and Mandryk, R. System Guidelines for Co-located, Collaborative Work on a Tabletop Display. In Proceedings of ECSCW 2003.

[11] Scott, S., Carpendale, S. and Habelski, S. Storage Bins: Mobile Storage for Collaborative Tabletop Displays. Computer Graphics and Applications, 25(4):58-65, 2005.

[12] Tang, C. \& Carpendale, S. An Observational Study on Information Flow during Nurses' Shift Work. In Proceedings of CHI 2007, pp. 219-228.

[13] Tang, C. \& Carpendale, S. Impacts of Technology Deployment on Information Assembly and Disassembly during Shift Change. In Workshop of Handover: Collaboration for Continuity of Work, ECSCW 2007.

[14] Vicente, K. The Human Factor. Vintage Canada Ed., 2004.

[15] Anoto: www.anoto.com accessed on March 27, 2008 